\title{
Influence of secondary flow corner vortex to boundary layer in a channel flow
}

Cite as: AIP Conference Proceedings 2118, 030011 (2019); https://doi.org/10.1063/1.5114739

Published Online: 27 June 2019

Daniel Duda, Jindřich Bém, Jiří Kovařík, Vitalii Yanovych, and Václav Uruba

ARTICLES YOU MAY BE INTERESTED IN

Reynolds number in laminar flows and in turbulence

AIP Conference Proceedings 2118, 020003 (2019); https://doi.org/10.1063/1.5114728

Sciences Committee and Reviewers: 38th Meeting of Departments of Fluid Mechanics and Thermodynamics

AIP Conference Proceedings 2118, 010002 (2019); https://doi.org/10.1063/1.5114725

Impact of the gas mass flow rate on orifice measurements accuracy

AIP Conference Proceedings 2118, 030008 (2019); https://doi.org/10.1063/1.5114736

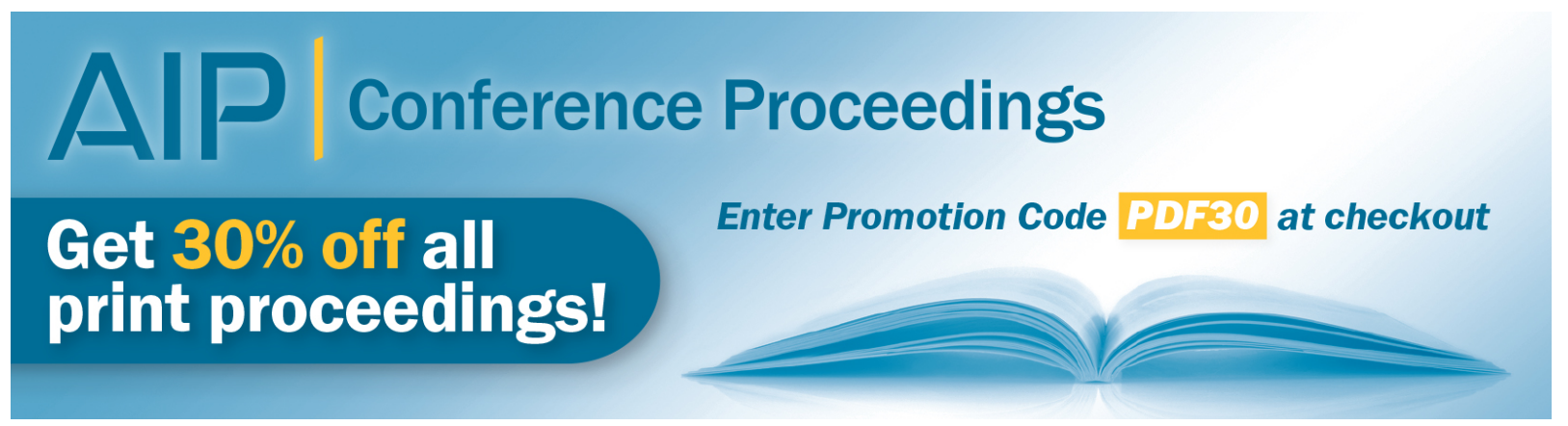




\title{
Influence of Secondary Flow Corner Vortex to Boundary Layer in a Channel Flow
}

\author{
Daniel Duda ${ }^{1, a)}$, Jindřich Bém ${ }^{1)}$, Jiří Kovařík ${ }^{1)}$, Vitalii Yanovych ${ }^{1)}$ and Václav \\ $\mathrm{Uruba}^{1,2, \mathrm{~b})}$ \\ ${ }^{1}$ Faculty of Mechanical Engineering, University of West Bohemia in Pilsen, Univerzitni 22, 30614 Pilsen, Czech \\ Republic, \\ ${ }^{2}$ Institute of Thermodynamics, Czech Academy of Sciences, Dolejškova 5, 182 00, Prague, Czech Republic. \\ ${ }^{a)}$ Corresponding author: dudad@kke.zcu.cz \\ b)uruba@kke.zcu.cz
}

\begin{abstract}
Boundary layer in developing channel flow of air is experimentally studied by using the Stereo Particle Image Velocimetry (PIV) technique. The measurement is performed at fixed distance $400 \mathrm{~mm}$ from the channel inlet and the Reynolds number (based on the channel length, i.e. the distance from the boundary layer origin) is controlled via the imposed velocity. Re ranges from $8 \cdot 10^{4}$ to $8 \cdot 10^{5}$. The displacement boundary layer thickness $\delta^{*}$ varies from 1.7 to 2.5 $\mathrm{mm}$ while the momentum one $\theta$ from 0.9 to $1.3 \mathrm{~mm}$. It is found, that the critical Reynolds number of transition to turbulence of the boundary layer is lowered by the vicinity of the other perpendicular wall of the square channel; more accurately - it is accelerated by the larger-scale secondary flow, which results into turbulence at slightly lower Reynolds numbers. The laminar-turbulent transition is first apparent on the profiles of the turbulent kinetic energy, later on the velocity profiles. The mechanism might be probably such, that the turbulent flow structures generated in the secondary flow in the corner via Richardson energy transfer mechanism migrate into the laminar boundary layer. While the largescale structures cannot feed from the limited-size boundary layer, the smaller ones can strengthen there.
\end{abstract}

\section{SECONDARY FLOW OF SECOND KIND}

The so called "secondary flow of second kind" in a channel of square or rectangular cross-section was first observed by Nikuradse [1] and has been studied almost an entire century both experimentally $[2,3]$ and theoretically $[4,5]$. The majority of these studies have been performed in a developed case, i.e. far from the beginning of the channel when the boundary layers meet. In that case, the observed secondary flow cells (or vortices) fill the entire cross-section of the channel and at large Reynolds numbers, there is a symmetric pair of vortices per corner, while at lower ones, there is only one vortex in a corner spontaneously breaking the symmetry [5].

Our motivation comes mainly from the turbomachinery [6], where the channel is short in comparison with its width and height; thus the flow is not fully developed. In addition, it is curved leading to centrifugal - first order secondary flows (the famous Görtler vortices [7], or Taylor vortices in case of rotating cylinder). Another example of secondary flow is the steady streaming - a steady component of oscillatory flow [8], whose existence has been experimentally proven even in superfluid helium [9], which is a quantum liquid with legendary properties.

\section{Description of Experiment}

We measure air flow in an empty measurement section of length $400 \mathrm{~mm}$ and cross-sectional size $125 \times 125 \mathrm{~mm}$ of open low-speed wind tunnel with maximum velocity up to $34 \mathrm{~m} / \mathrm{s}$. By using the Stereo Particle Image Velocimetry (PIV) technique $[10,11]$ we measure all three components of velocity in a small area at the open end of the tunnel. Two cameras FlowSense MkII for Stereo PIV are inclined symmetrically with angle $45^{\circ}$ (more sensitive 
to the weak in-plane motions than the usual $90^{\circ}$ setup). The observed area has approximate size $24 \times 22 \mathrm{~mm}$, the grid resolution is $0.47 \mathrm{~mm}$. Double frame method is used, the interval depends on imposed velocity $(1.7-34 \mathrm{~m} / \mathrm{s})$ and ranges from 13 to $250 \mu \mathrm{s}$. The time between frame pairs is $135 \mathrm{~ms}$, therefore the instantaneous velocity fields can be considered to be statistically independent.

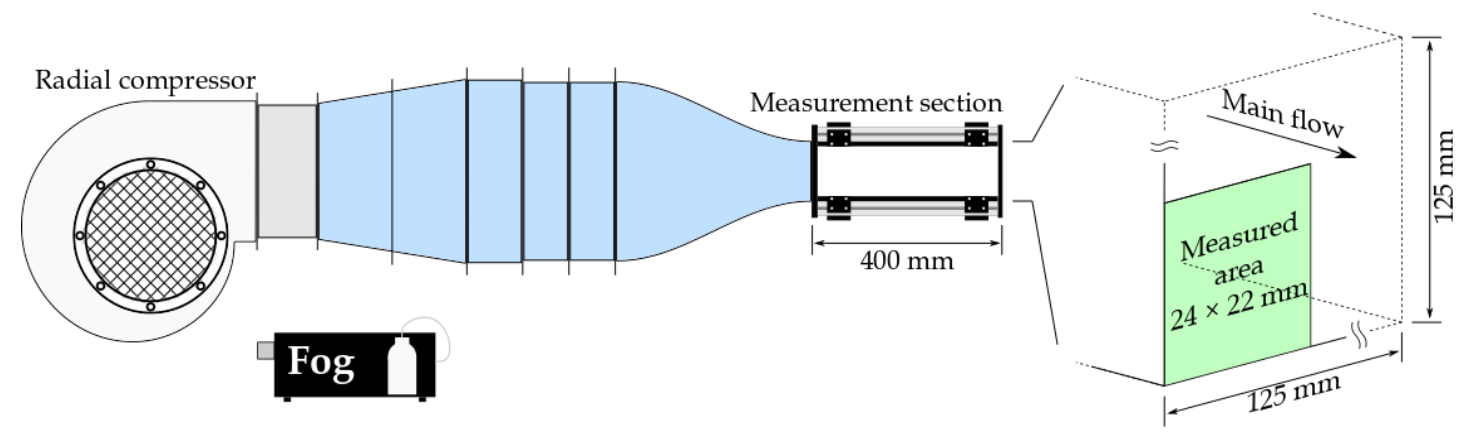

FIGURE 1. Sketch of the experimental setup. Left part shows the low-speed wind tunnel. The droplets produced by fog generator Safex are sucked to the inlet of tunnel. The test section is empty and the secondary flow in the corner can develop along its length. Right part shows schematically (not to scale) the positioning of the measured area of the Stereo PIV at the open end of the test section. The studied area is perpendicular to the main flow and covers approximately $24 \times 22 \mathrm{~mm}$.

\section{Reynolds Number}

The usual definition of Reynolds number used in the literature is based on the hydraulic diameter [2] of the channel. In all this cases, the flow was fully developed, i.e. there is no more the potential core and the boundary layer fills the entire cross-section. In our case the situation is different - the channel is short compared with its width (length $=3.2 \times$ the width), because this is more similar to an idealized inter-blade channel in a turbine.

The length dimension, which is physically relevant in our case is the thickness of the boundary layer, because this size naturally limits the size of vortices. The boundary layer thickness itself is a measured quantity - kind of a result, and therefore even in the boundary layer studies [11] the dimension used for defining Reynolds number is the distance from origin of the boundary layer. Therefore, we prefer to use this definition in our case as well.

\section{Boundary Layer Thickness}

There are multiple definitions of boundary layer thickness, here we use two of them: the displacement boundary layer thickness $\delta^{*}$ defined as

$$
\delta^{*}=\int_{0}^{\infty}\left(1-\frac{w(x)}{w_{\mathrm{far}}}\right) \mathrm{d} x \approx \sum_{i=0}^{N_{x}}\left(1-\frac{w(i \cdot \Delta x)}{w\left(N_{x} \cdot \Delta x\right)}\right) \Delta x,
$$

where $w$ is the ensemble averaged streamwise velocity at distance $x$ from the wall, $w_{\text {far }}$ is its value far enough in the potential core. The ratio $w(x) / w_{\text {far }}$ as a function of $x$ is shown in Fig. $2 \mathrm{~b}$. As our measurement is performed only at grid points spaced by $\Delta x=0.47 \mathrm{~mm}$, we had to approximate integration by summation.

The momentum thickness $\theta$ can be estimated accordingly:

$$
\theta=\int_{0}^{\infty} \frac{w(x)}{w_{\mathrm{far}}}\left(1-\frac{w(x)}{w_{\mathrm{far}}}\right) \mathrm{d} x \approx \sum_{i=0}^{N_{x}} \frac{w(i \cdot \Delta x)}{w\left(N_{\chi} \cdot \Delta x\right)}\left(1-\frac{w(i \cdot \Delta x)}{w\left(N_{x} \cdot \Delta x\right)}\right) \Delta x
$$

Boundary layer thicknesses for different Reynolds numbers are shown in Fig. 2c; Fig 2d shows the shape factor, which would achieve the value of 2.59 for laminar (Blasius) boundary layer, or 1.4 for turbulent boundary layer. We do not fit these classical values, probably due to the discretization with too large step. This parameter suggests transition to turbulence at $R e_{L} \sim 1.3 \cdot 10^{5}$, which is apparent in Fig. $2 \mathrm{~b}$ as the yellow line "between" the two type bunches. 
(a) $R e_{L}=8 \cdot 10^{4}$

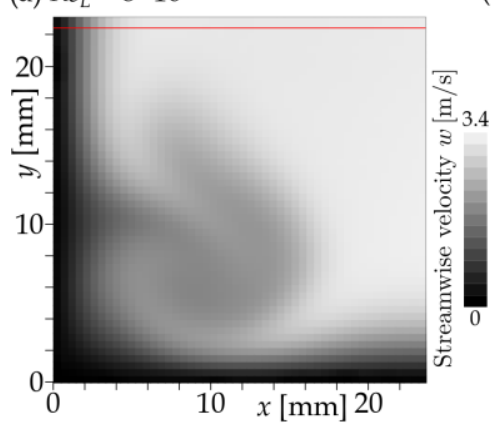

(b)

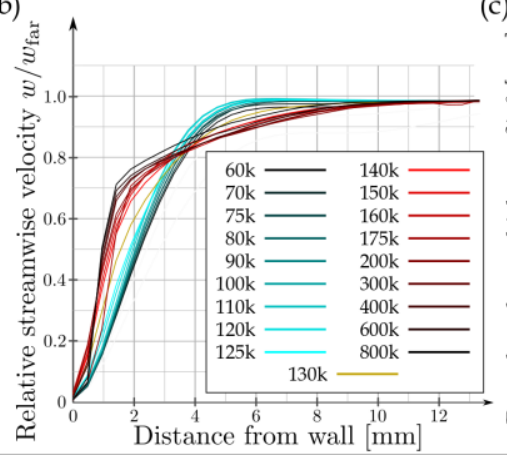

(c)

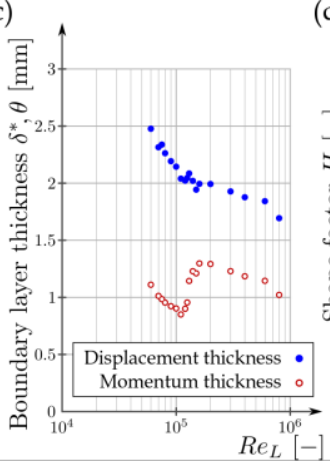

(d)

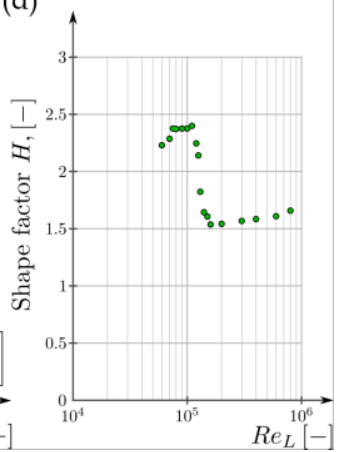

FIGURE 2. (a) Example of an averaged velocity field (only the streamwise component is shown in grayscale) measured at Reynolds number $R e_{L}=8 \cdot 10^{4}$. Note the low-momentum material ejected from the wall by the corner vortex. Red line in the upper part shows the position of velocity profiles shown in (b). (b) the velocity profiles of average streamwise velocities for Reynolds numbers $\operatorname{Re}_{L}$ ranging from $6 \cdot 10^{4}$ to $8 \cdot 10^{5}$ shown in color, "k" plays for " $10^{3}$ ". (c) Boundary layer thicknesses as a function of $R_{L}$ determined from the velocity profiles shown in (b). (d) Shape factor of the boundary layer, $H=\delta^{*} / \theta$.

\section{Transition to Turbulence}

Boundary layer far $(22 \mathrm{~mm})$ from the end wall transits into turbulence at $R e_{L} \sim 1.3 \cdot 10^{5}$; however, this is a consequence of processes in the corner vortex, which transits at lower $R_{L}$. The scenario we observed is that first the large-scale laminar secondary flow vortex induces smaller vortices in the strong shear region, see Fig. 5a and 6a, their position is not stable and at $\operatorname{Re}_{L} \sim 1.1 \cdot 10^{5}$ (Fig. $3 \mathrm{c}$ and $4 \mathrm{c}$ ) they start to migrate into the boundary layer, where only the structures of appropriate size can earn energy from the laminar shear. At even larger $R e_{L}$, the influence opposes - structures from turbulent boundary layer overlay the laminar corner vortex. The structure of secondary flow changes to the turbulent regime of pair of smaller counter-rotating vortices, as it has been previously suggested by Uhlmann [5] in fully developed channel flow in a numerical simulation.

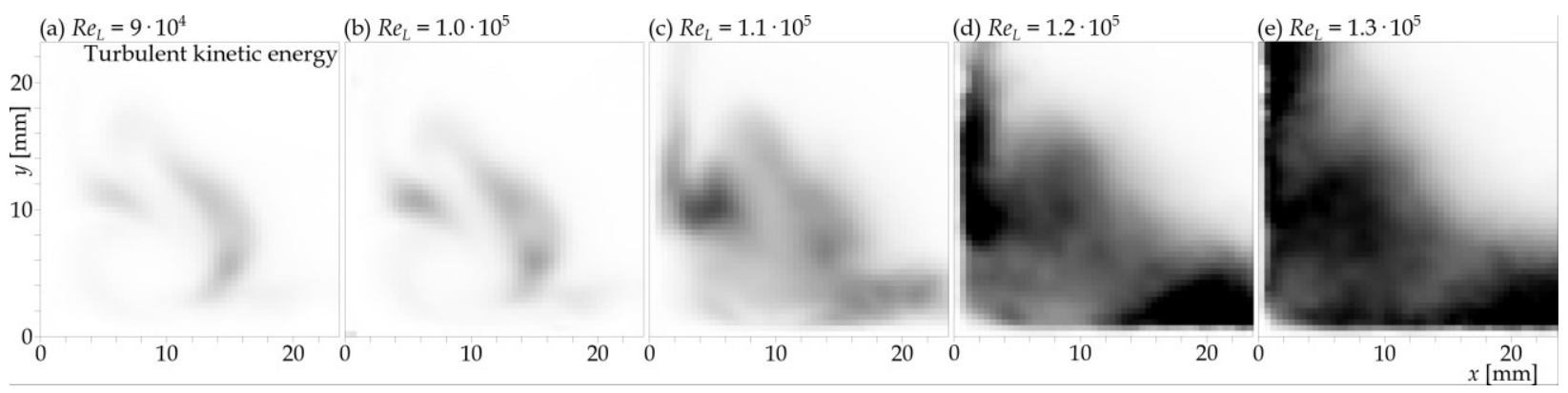

FIGURE 3. Spatial map of the turbulent kinetic energy of the ensemble at different Reynolds numbers $\operatorname{Re}_{L}:$ (a) $\operatorname{Re}_{L}=9 \cdot 10^{4}$, (b) $R e_{L}=1.0 \cdot 10^{5}$, (c) $R e_{L}=1.1 \cdot 10^{5}$, (d) $R e_{L}=1.2 \cdot 10^{5}$ and (e) $R e_{L}=1.3 \cdot 10^{5}$. The gray-scale is normalized by $\operatorname{Re}_{L}{ }^{2}$
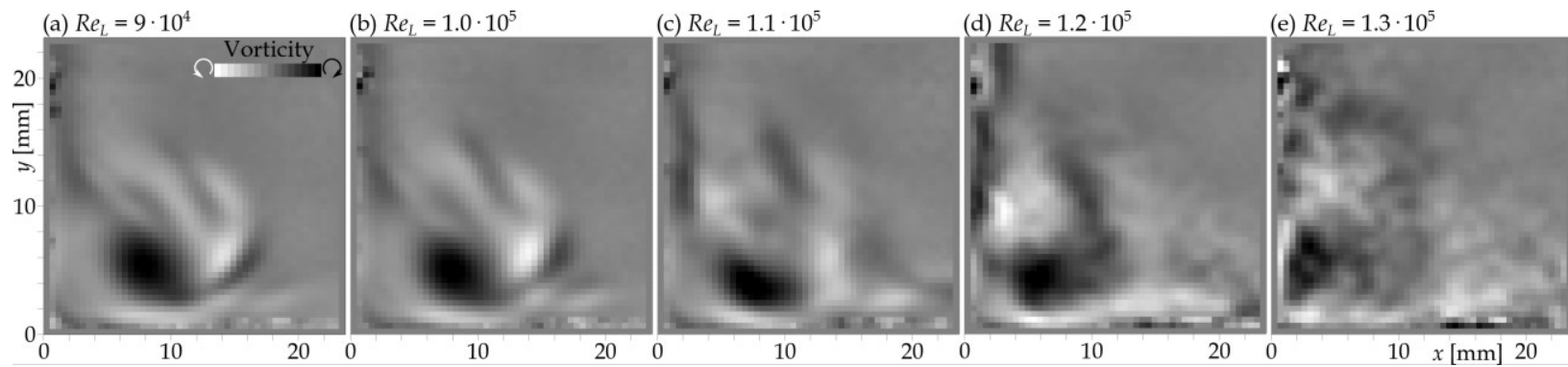

FIGURE 4. Spatial map of the averaged in-plane vorticity at different Reynolds numbers $\operatorname{Re}_{L}$ : (a) $\operatorname{Re}_{L}=9 \cdot 10^{4}$, (b) $\operatorname{Re}_{L}=1.0 \cdot 10^{5}$, (c) $R e_{L}=1.1 \cdot 10^{5}$, (d) $R e_{L}=1.2 \cdot 10^{5}$ and (e) $R e_{L}=1.3 \cdot 10^{5}$. The gray-scale is normalized by $R e_{L}$, dark colors represent clock-wise spin, light ones anti-clock-wise spin in the studied plane. 
The mentioned hypothesis is supported by Fig. 5, where it is displayed the turbulent kinetic energy of the transition case of $R e_{L}=1.1 \cdot 10^{5}$ of different length-scales $[13,14]$. At largest length-scales we see fluctuations only in the secondary flow corner vortex (Fig. 5a), at middle scales (Fig. 5c) fluctuations are located in the nearby boundary layer regions as well, while at smallest scales (Fig. 5e) the fluctuations in the boundary layers are even stronger than those in corner vortex.

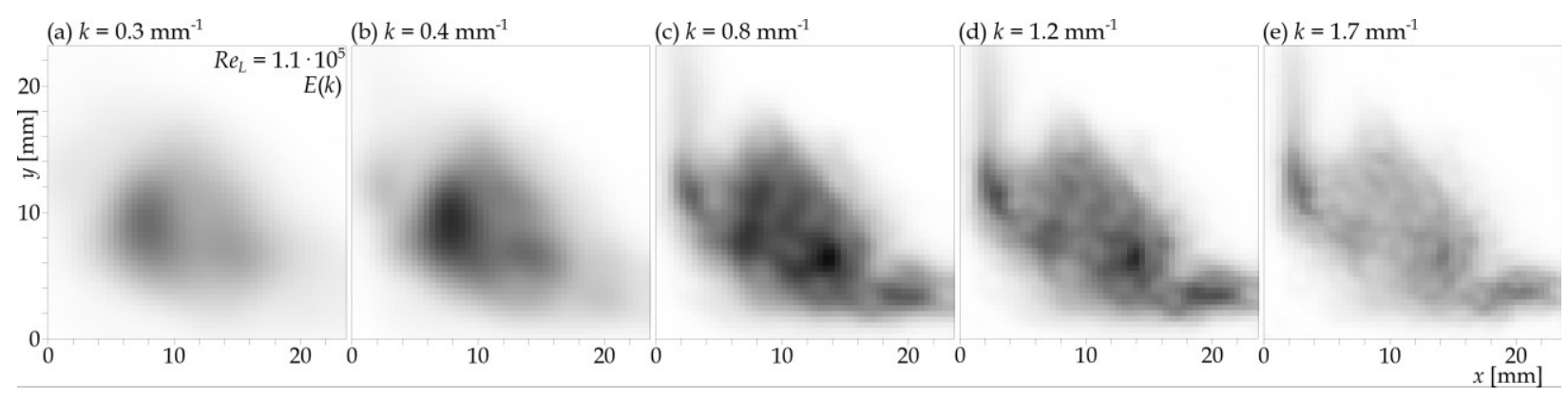

FIGURE 5. Spatial map of the turbulent kinetic energy at different length-scales at $\operatorname{Re}_{L}=1.1 \cdot 10^{5}$ (see Fig. 3c and $4 \mathrm{c}$ ). The energy spectral density is normalized by $k^{-5 / 3}, k=1 / \sigma$ is the wave number, $\sigma$ the size band-pass filter used for exctracting the size-dependent component of the in-plane velocity field [13].

We can roughly conclude that the boundary layer has been "infected" with turbulence, and, as the boundary layer is a good environment for turbulence, it can strengthen there enough to change the overall character of secondary flow, which is apparent when comparing Fig. $4 \mathrm{a}$ and $4 \mathrm{e}$.

\section{ACKNOWLEDGMENTS}

The work was supported from ERDF under project "Research Cooperation for Higher Efficiency and Reliability of Blade Machines (LoStr)" No. CZ.02.1.01/0.0/0.0/16_026/0008389.

\section{REFERENCES}

1. J. Nikuradse, "Untersuchungen über die Geschwindigkeitsverteilung in turbulenten Strijmungen", Ph.D. thesis, Götingen, 1926.

2. H. Fujita, H. Yokosawa and M. Hirota, Secondary flow of the second kind in rectangular ducts with one rough wall, Experimental Thermal and Fluid Science 2:72-80 (1989).

3. V. Uruba, O. Hladík and P. Jonáš, Dynamics of secondary vortices in turbulent channel flow, Journal of Physics: Conference Series 318 (6), 062021 (2011).

4. A. Bottaro, H. Soueid and B. Galletti, Formation of secondary vortices in turbulent square-duct flow, AIAA Journal 44 (4), 803-811 (2006).

5. M. Uhlmann, A. Pinelli, G. Kawara and A. Sekimoto, Marginally turbulent flow in a square duct, J. Fluid Mech. 588: 153-162 (2007).

6. G. Ilieva A deep insight to secondary flows, Defect and Diffusion Forum 379: 83-107 (2017).

7. W. S. Saric, Görtler vortices Annual Review of Fluid Mechanics 26 (1), 379-409 (1994).

8. K. Chong, S. D. Kelly, S. T. Smith and J. D. Eldredge, Transport of inertial particles by viscous streaming in arrays of oscillating probes, Physical Review E 93 (1) 013109 (2016).

9. D. Duda, M. La Mantia and L. Skrbek, Streaming flow due to a quartz tuning fork oscillating in normal and superfluid He 4, Physical Review B 96 (2) 024519 (2017).

10. C. Tropea, A. L. Yarin, and J. F. Foss, "Springer handbook of experimental fluid mechanics", Springer (2007)

11. D. Jašíková, M. Kotek and V. Kopecký, An effect of entrance length on development of velocity profile in channel of millimeter dimensions, AIP Conference Proceedings 1745, 020018 (2016).

12. A. Agrawal, Measurement of spectrum with particle image velocimetry, Experiments in fluids 39 (5): 836-840 (2005).

13. D. Duda and V. Uruba, PIV of air flow over a step and discussion of fluctuation decompositions, AIP Conference proceedings 2000, 020005 (2018). 